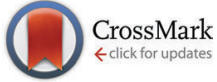

Cite this: Phys. Chem. Chem. Phys., 2016, 18, 19588

Received 12th March 2016,

Accepted 9th June 2016

DOI: $10.1039 / c 6 c p 01698 g$

www.rsc.org/pccp

\title{
Spectroscopy and microscopy of single molecules in nanoscopic channels: spectral behavior vs. confinement depth
}

\author{
Benjamin Gmeiner, ${ }^{a}$ Andreas Maser, ${ }^{a b}$ Tobias Utikal, $^{a}$ Stephan Götzinger ${ }^{a b c}$ and \\ Vahid Sandoghdar*ab
}

\begin{abstract}
We perform high-resolution spectroscopy and localization microscopy to study single dye molecules confined to nanoscopic dimensions in one direction. We provide the fabrication details of our nanoscopic glass channels and the procedure for filling them with organic matrices. Optical data on hundreds of molecules in different channel depths show a clear trend from narrow stable lines in deep channels to broader linewidths in ultrathin matrices. In addition, we observe a steady blue shift of the center of the inhomogeneous band as the channels become thinner. Furthermore, we use super-resolution localization microscopy to correlate the positions and orientations of the individual dye molecules with the lateral landscape of the organic matrix, including cracks and strain-induced dislocations. Our results and methodology are useful for a number of studies in various fields such as physical chemistry, solid-state spectroscopy, and quantum nano-optics.
\end{abstract}

\section{Introduction}

Cryogenic spectroscopy of single molecules ${ }^{1-3}$ opened the way to high-resolution spectral and spatial investigations of quantum emitters such as semiconductor quantum dots, ${ }^{4,5}$ color centers ${ }^{6}$ and rare earth ions ${ }^{7}$ in solid matrices. In the pioneering efforts of the late 1980s and early 1990s, it was shown that at temperatures below about $2 \mathrm{~K}$ the zero-phonon lines (ZPL) of aromatic molecules such as pentacene or terrylene can become as narrow as a few tens of $\mathrm{MHz}$, corresponding to Fourier-transform-limited values expected from the excited-state lifetimes of a few nanoseconds. Shortly after the advent of this methodology, a number of experiments studied the response of the ZPLs to external influences such as pressure and electric field as well as internal conditions like the nature of the host material, e.g., whether it is crystalline or amorphous. ${ }^{3}$ In particular, spectral jumps and fluctuations could be directly observed. ${ }^{8}$ Spectral instabilities had been known in solidstate spectroscopy for several decades and are understood to be caused by changes in the environment that lead to variations of the local fields at the position of the guest emitter.

\footnotetext{
${ }^{a}$ Max Planck Institute for the Science of Light, 91058 Erlangen, Germany. E-mail: vahid.sandoghdar@mpl.mpg.de

${ }^{b}$ Department of Physics, Friedrich Alexander University (FAU) Erlangen-Nürnberg, 91058 Erlangen, Germany

${ }^{c}$ School of Advanced Optical Technologies (SAOT), Friedrich Alexander University (FAU) Erlangen-Nürnberg, 91058 Erlangen, Germany
}

However, their direct visualization became only possible through single molecule spectroscopy.

Spectral diffusion, fluctuations and jumps are ubiquitous phenomena in systems such as molecules, rare earth ions, semiconductor quantum dots or color centers, but the details of their microscopic origins can vary. ${ }^{9}$ The jump events are often discussed in the context of quantum mechanical tunneling between two states of a so-called two-level system. ${ }^{3}$ In some cases, such a tunneling can be influenced by an external electric field, ${ }^{10-12}$ mechanical stimulus, ${ }^{13}$ or light fields. ${ }^{14}$ In the case of slow spectral jumps of terrylene in para-terphenyl, it was shown theoretically that the rotation of bonds in the matrix molecules can cause spectral jumps. ${ }^{15}$

In general, spectral instabilities are produced by defects and dynamics in the host matrix, e.g., caused by imperfect crystallization at interfaces. Indeed, self-assembled quantum dots are known to need about 50 nanometer of a capping layer to yield a stable luminescence. ${ }^{16}$ Similarly, broad lines and unstable resonances have been reported for nanocrystal hosts. ${ }^{17}$ In this article, we report on measurements that provide new insight into the behavior of the molecular spectra in crystalline matrices formed under geometric confinement in one dimension. Our strategy is to prepare samples of various thickness in submicrometer deep channels and collect statistics about their spatio-spectral behavior. In addition to their fundamental interest, thin crystalline samples are also desirable for a number of quantum optical experiments. 


\section{Experimental}

\subsection{Sample preparation}

Fig. $1 \mathrm{a}$ and $\mathrm{b}$ illustrate the procedure for preparing a nanochannel. We employ standard grayscale photolithography and a subsequent reactive ion etching step in a fused silica cover glass to create an array of $100 \mu \mathrm{m} \times 100 \mu \mathrm{m}$ fields with varying depths, ranging from $60 \mathrm{~nm}$ to $730 \mathrm{~nm}$. This structured cover glass and a small fused silica substrate are then cleaned and pre-bonded at room temperature by bringing them into optical contact, where they are drawn together by van der Waals forces. ${ }^{18}$ Subsequent annealing of the assembly at $950{ }^{\circ} \mathrm{C}$ for several hours in a muffle kiln operating at atmospheric pressure converts the pre-bond into a strong fusion bond. ${ }^{19}$ Fig. 1c shows a white-light microscope image of a bonded nanochannel with $5 \times 5$ fields. The individual domains in the middle can be distinguished by thin film interference. The dark regions at the left and right corners are the bonded areas.

To fill the channel, we placed a small naphthalene crystal doped with dibenzanthanthrene (DBATT) molecules (concentration about $10^{-6} \mathrm{M}$ ) at one entrance of the channel (see Fig. 1d). Next, the whole sample was heated by a Peltier element until the naphthalene melted and was drawn into the nanochannel by capillary forces. ${ }^{20}$ We then switched off the Peltier element, and the sample crystallized (see Fig. 1e). In this manner, we could form doped naphthalene crystals with thicknesses between $60 \mathrm{~nm}$ and $730 \mathrm{~nm}$ in a single crystallization process and on the same substrate. Fig. If displays a cross-polarized microscope image of the final sample. Cracks which are produced during the crystallization process run through the whole sample.

The sample was transferred into a cryostat (CryoVac) operating at superfluid helium temperature. In Fig. 2, we show examples of six cross-polarized white-light images of naphthalene crystals
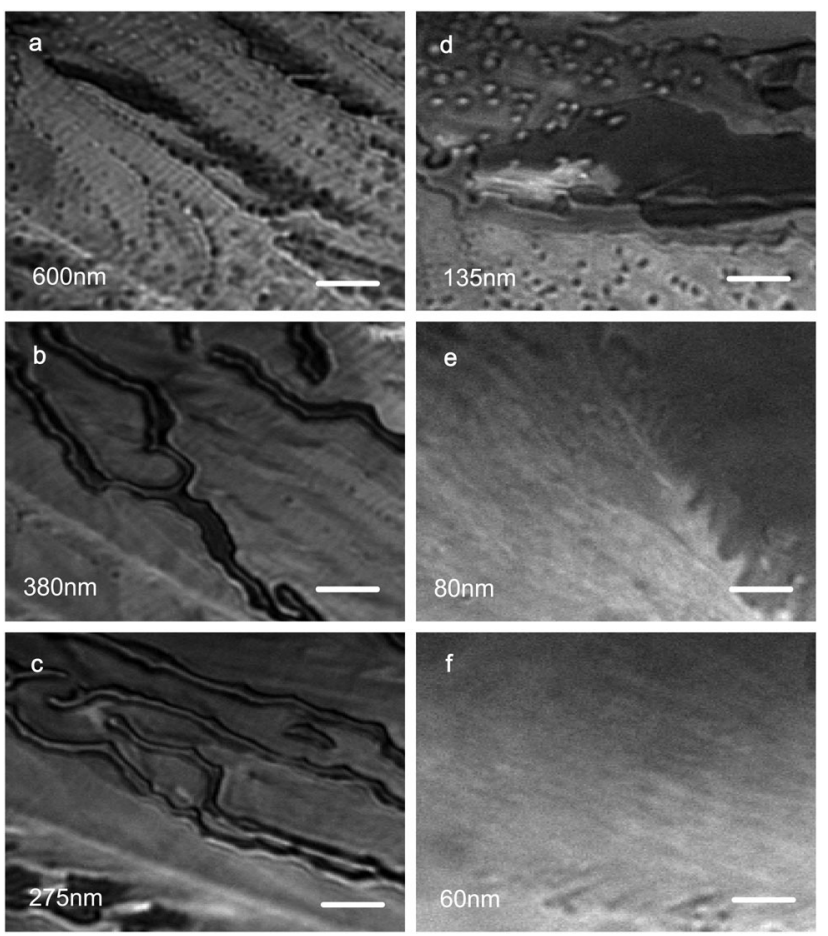

Fig. 2 Cross-polarized white light images of the naphthalene crystal in the cryostat. As the thickness is reduced from (a) to (f), fewer cracks and periodic strain-induced dislocations are visible. The thickness for each crystal is written in the left corner. The scale bar indicates $10 \mu \mathrm{m}$. See text for details.

with different thicknesses recorded at $1.5 \mathrm{~K}$. The different grey values of crystal domains are caused by the birefringence of naphthalene. The interaction of naphthalene molecules with the silica surface and the subsequent change of temperature
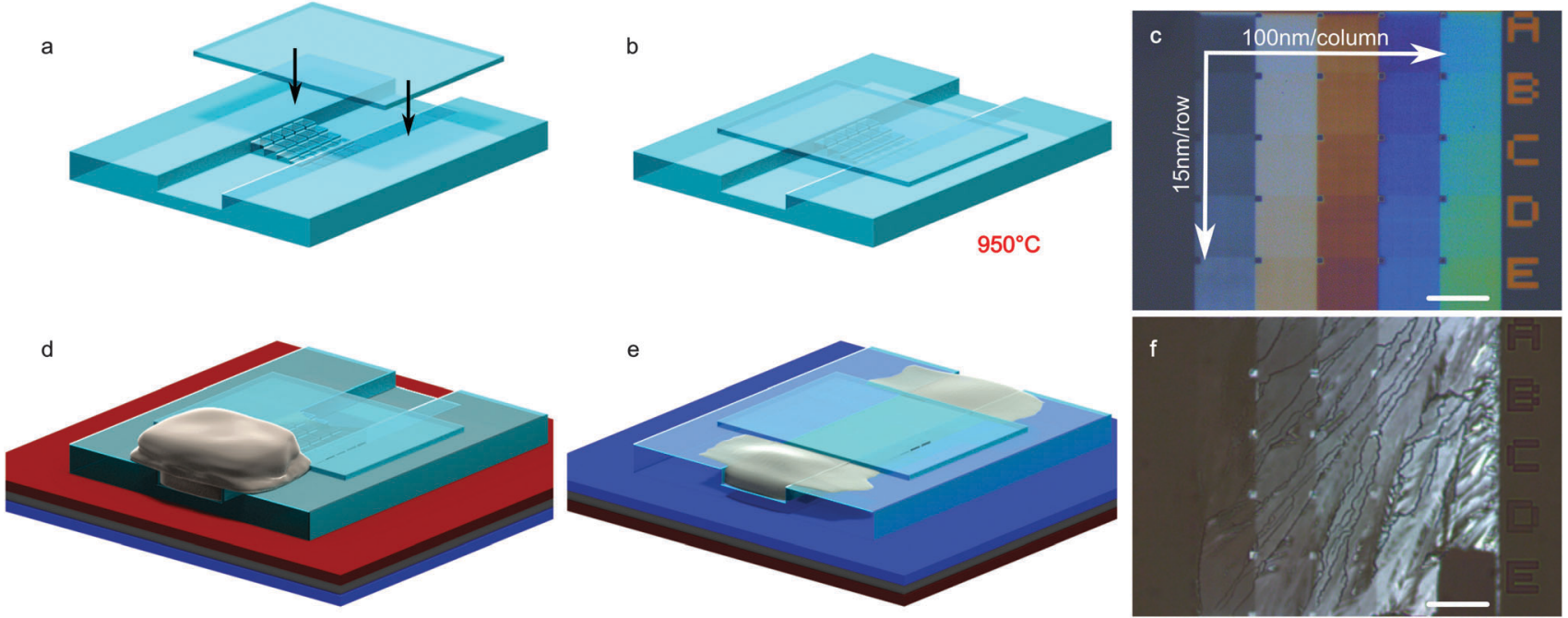

Fig. 1 Sample preparation: (a) a cover glass with a structured trench and a small lid are pre-bonded at room temperature. The assembly forms a nanochannel with an array of different depths. (b) The sample is annealed at $950{ }^{\circ} \mathrm{C}$ in a muffle kiln, producing a fusion bond. (c) White-light image of a bonded nanochannel. (d) A small doped naphthalene crystal is placed at the channel exit, and the whole configuration is heated by a Peltier element. The molten naphthalene is sucked into the nanochannel. (e) Cooling the sample crystallizes the naphthalene. (f) Cross-polarized microscope image of the naphthalene crystal inside the nanochannel. Scale bars indicate $100 \mu \mathrm{m}$. 
strain the crystal, resulting in further cracks (see Fig. 2b and c). These cracks usually arise between crystal domains, where the strain can be released most easily.

Upon closer scrutiny, one also notes periodic parallel structures for thicker regions. We attribute this effect to a slip phenomenon, where the naphthalene crystal releases its strain during the cool-down process. ${ }^{21,22}$ Dislocations move under strain, add up, and lead to periodic plastic deformation along crystallographic planes. ${ }^{23,24}$ As the crystal thickness is decreased, fewer cracks are visible and the periodic dislocations seem to vanish. In general, the crystal releases its strain by the formation of cracks, however, here it also sticks to both sides of the glass. If the channel is only tens of nanometers, the closeby interfaces counteract the formation of cracks.

\subsection{Optical setup}

Fig. 3 sketches our experimental setup. We used a tunable, cw, single-mode dye laser (Coherent 899-29 Auto Scan) with a linewidth of about $2 \mathrm{MHz}$ to excite the narrow transitions of DBATT molecules. The laser intensity was stabilized by using a feedbacked acousto-optical modulator. Insertion of a lens in the excitation path allowed for switching between wide-field and confocal illumination. A single aspheric lens (0.55 NA) focussed the light down to a spot with full width at halfmaximum (FWHM) of $630 \mathrm{~nm}$. The sample and the lens were moved in three dimensions with a piezo slider system for focusing and addressing the individual crystal domains. Within a single field, we could also laterally scan the laser spot with a galvanometric mirror system placed in a conjugated back focal plane of the aspheric lens.

By scanning the laser frequency through the inhomogeneous absorption band of DBATT, we probed individual molecules as they became resonant with the laser and fluoresced. ${ }^{2}$ The emitted red-shifted fluorescence was measured with either a single-photon counting module (SPCM) (PerkinElmer), or a charged-coupled device (CCD) camera (Hamamatsu, Orca-R2). Here, the laser frequency was scanned in $10 \mathrm{MHz}$ steps, where each new frequency triggered the exposure of a frame at the CCD camera. The measurements recorded at different excitation frequencies yielded a stack of image frames, which were then

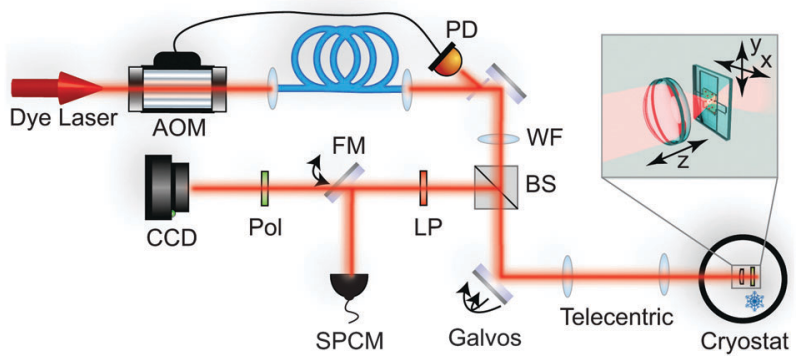

Fig. 3 Schematic of the experimental setup. A tunable single-mode dye laser is focused onto the naphthalene crystal inside a cryostat. Fluorescence is detected by either a CCD camera or a single-photon counting module (SPCM). AOM: acousto-optical modulator; PD: photodiode; WF: wide-field lens; Pol: polarizer; FM: flip mirror; BS: beam splitter; LP: longpass filter. analyzed to extract the spectral line shape, transition frequency and position of the examined molecules.

\section{Localization of single molecules}

A great advantage of high-resolution cryogenic spectroscopy is that it provides a straightforward means to tag each molecule through its resonance frequency. If the doping level is not too high, individual molecules can be excited one at a time by tuning the laser frequency. As a result, one can localize the center of the molecule's point-spread function (PSF) to within a few nanometers. ${ }^{25}$ In this fashion, the spectral behavior of single molecules can be correlated with their orientation and location within a specific environment. ${ }^{26,27}$

In our setup, we reached a localization precision of about $20 \mathrm{~nm}$ without taking any measures against mechanical vibration or drift. A cross-polarized white-light image of a $730 \mathrm{~nm}$ thick naphthalene crystal is shown in Fig. 4a together with the locations of over 16000 DBATT molecules. The correlation of the two images reveal that DBATT molecules are often embedded at cracks and dislocations. This is consistent with the point of view that the impurity DBATT molecules are pushed out of the naphthalene matrix during the cool-down process. ${ }^{28}$ We remark that we expect the influence of a crack on the localization precision to be negligible. Such an effect would result in an asymmetry (elongation) of the PSF, but the PSFs recorded for the data shown did not suffer from this effect (see, e.g., inset (e) in Fig. 4).

To learn about the orientation of the individual molecules, we employed circularly polarized light and placed orthogonal polarizers in front of the camera in two successive scans. In Fig. $4 \mathrm{~b}$, the dipole orientations of localized molecules are depicted by arrows. We find that the in-plane projection of the individual dipoles are predominately aligned parallel to each other within a single crystal domain. Furthermore, most are oriented parallel to the observed slip lines. As the slip plane of naphthalene is usually (001), we assume that the molecules are oriented parallel to this crystal plane. ${ }^{21,22}$

Fig. 4c shows a $63 \mathrm{~nm}$ thin crystal with localized molecules. Here, no dislocations can be seen and the molecules seem to be distributed homogeneously. As in the case of the $730 \mathrm{~nm}$ thick crystal, the in-plane projection of the individual dipoles were predominately aligned parallel to each other.

\section{Strain-induced shift of the inhomogeneous band}

Fig. 5a shows a fluorescence excitation spectrum recorded as the laser frequency was scanned across the inhomogeneous absorption band of the DBATT-naphthalene sample. The measurement was performed in a confocal configuration on a $730 \mathrm{~nm}$ thick crystal. The distribution of the resonances over more than two nanometers is much larger than what is known from sublimated crystals. $^{29}$ This behavior was also observed in nanocapillaries ${ }^{20}$ and points to the heterogeneity of the crystal environment. 

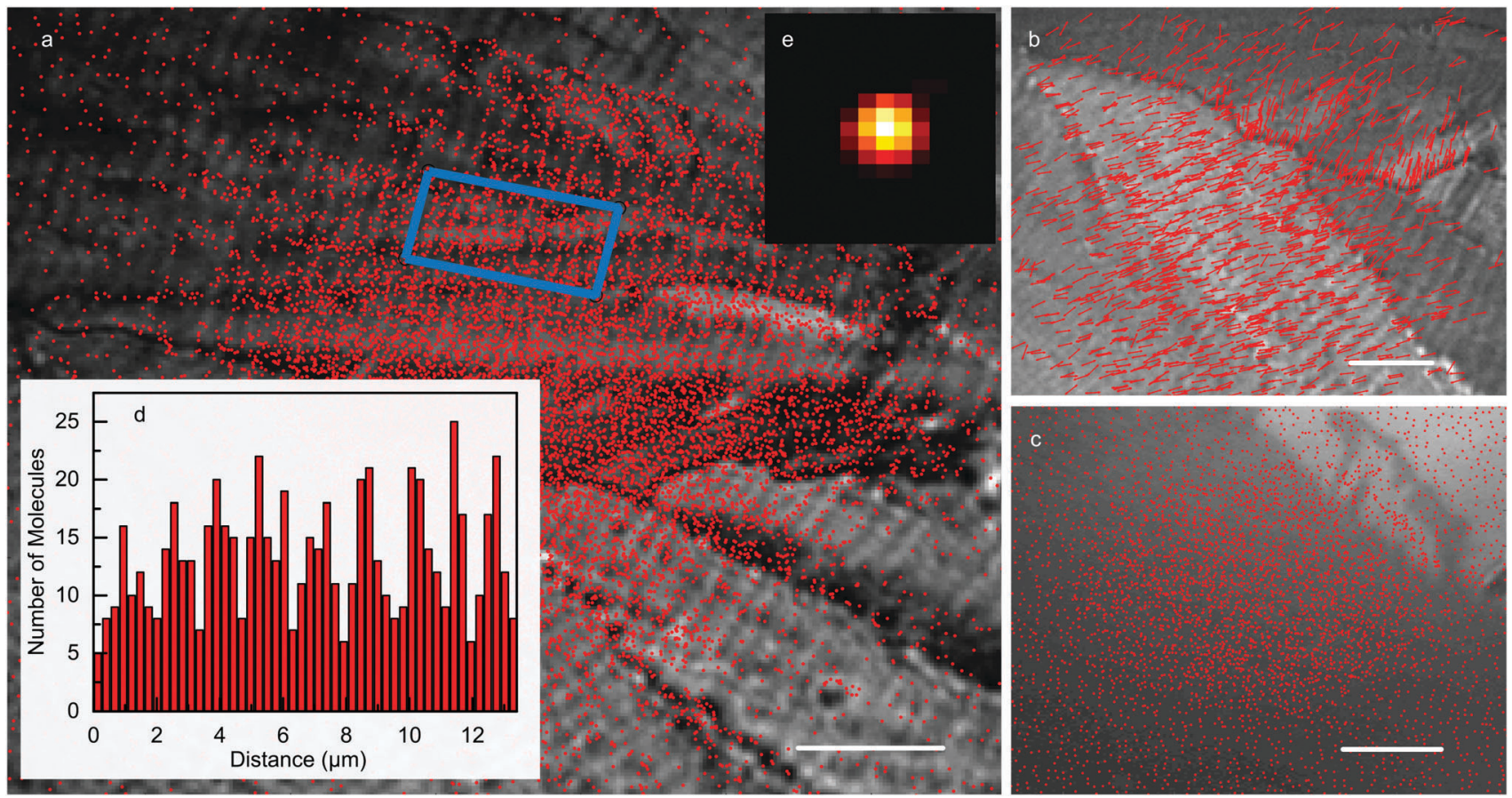

Fig. 4 (a) Cross-polarized white-light image of a $730 \mathrm{~nm}$ thick naphthalene crystal superposed with localized DBATT molecules. (b) Dipole orientations of localized single molecules are depicted by arrows. (c) Localized molecules in a $63 \mathrm{~nm}$ thick naphthalene crystal. Scale bars indicate $10 \mu \mathrm{m}$. Inset (d) shows the distribution of molecules in the marked area of (a) after binning and adding the signals along the long side. The periodicity of the molecular population is now quite evident. Inset (e) shows exemplarily a recorded PSF with a FWHM of $630 \mathrm{~nm}$.
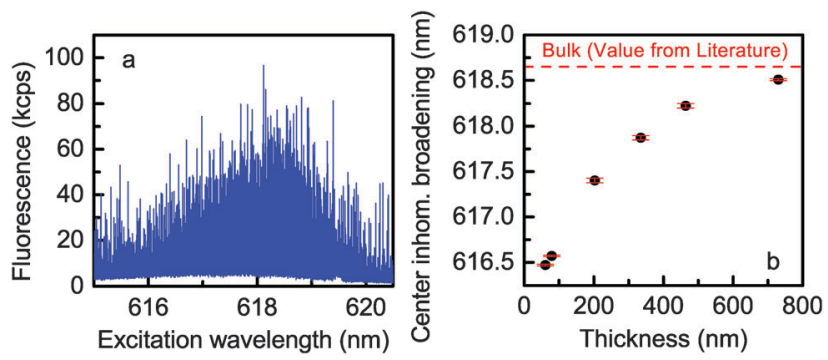

Fig. 5 (a) Fluorescence excitation spectrum of single-molecule ZPLs in a channel with a depth of $730 \mathrm{~nm}$. (b) The center of the absorption bands as a function of the channel thickness.

The large spectral breadth can be advantageous for experiments in hybrid quantum optics, e.g. coupling molecules to alkali atoms. ${ }^{30}$

To examine the behavior of the inhomogeneous band in various channel depths, we recorded fluorescence excitation spectra of single molecules in small regions using wide-field illumination and SPCM detection. The centers of these distributions are plotted as a function of crystal thickness in Fig. 5b. We find that the maximum of the inhomogeneous distribution tends towards higher energies as the crystals become thinner, whereby at the maximum thickness of $730 \mathrm{~nm}$, almost the literature bulk value ${ }^{29}$ of $618.65 \mathrm{~nm}$ is reached.

To gain further insight into the subdomains of each crystal field, we recorded a wide-field fluorescence excitation spectrum with a CCD camera. We then extracted the center of the inhomogeneous broadening for every pixel, using the same
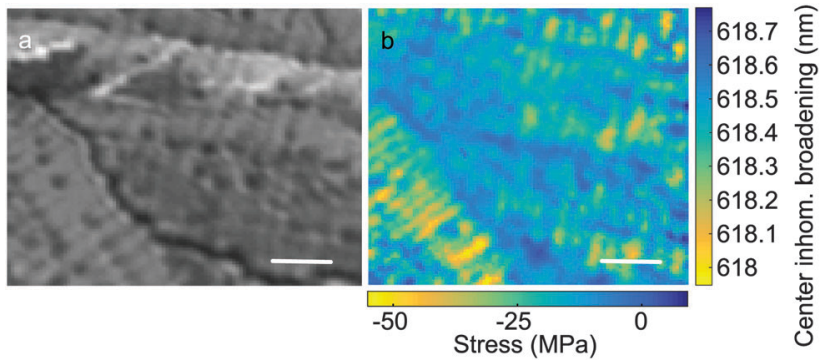

Fig. 6 (a) Cross-polarized white-light image of a $730 \mathrm{~nm}$ thick naphthalene crystal inside the cryostat. (b) The center of the inhomogeneous broadening plotted for each pixel in (a), using the color code shown on the right. Scale bar indicates $5 \mu \mathrm{m}$.

analysis as before. Fig. 6a shows a cross-polarized white-light image of a region of the $730 \mathrm{~nm}$ thick crystal. In Fig. 6b, we display the center of the inhomogeneous broadening at each pixel, revealing a clear correlation with the sub-micrometer crystal domains.

Early single-molecule spectroscopy experiments showed that the resonance frequencies of molecules are very sensitive to mechanical stress and strain. In particular, it was demonstrated that stress in the host crystal induced by hydrostatic pressure causes a strong red shift ${ }^{31-33}$ in line with the established knowledge of the solvent shift model. ${ }^{34}$ It has been shown experimentally that a linear approximation for the resonance frequency shift as function of pressure is justified for pressures up to $3.5 \mathrm{GPa} .{ }^{35}$ We now compare the internal strain of the various 
crystal thicknesses in our sample to gain further information on the crystallization process of naphthalene under confinement.

Using the volume expansion of $\varepsilon(300 \mathrm{~K})=0.06$, which relates the volume $V$ at a certain temperature to the volume $V_{0}$ at $0 \mathrm{~K}$ via the relation $V(T)=(1+\varepsilon(T)) V_{0},{ }^{36,37}$ naphthalene contracts by about $6 \%$ when it is cooled down to $1.5 \mathrm{~K}$. If this contraction is prohibited due to a rigid confinement, the crystal is strained. Taking the compressibility of $K=7.2 \times 10^{-11} \mathrm{~Pa}^{-1}$ for naphthalene, ${ }^{38}$ this strain corresponds to an external pressure of -830 MPa. Former studies with similar systems (i.e. pentacene in $p$-terphenyl and terrylene in $p$-terphenyl) showed resonance shifts of about $1 \mathrm{MHz} \mathrm{hPa}{ }^{-1} \cdot{ }^{31-33}$ Using these values and assuming no relaxation, we would expect the maximal shift to be in the range of about $7 \mathrm{THz}$. If we extrapolate our observed shifts for the crystal thickness approaching $0 \mathrm{~nm}$, we expect a shift of about 2 THz. The general good agreement of this estimate with the value of $7 \mathrm{THz}$ discussed above is consistent with the assumption that a one-dimensional confinement prohibits the relaxation of naphthalene and, thus, strains the crystal. This hypothesis is also in line with the observation that fewer cracks and slip dislocations take place in thinner fields (see Fig. 2).

Assuming a resonance shift of $1 \mathrm{MHz} \mathrm{hPa}^{-1}$ and a fitting accuracy of $4 \mathrm{GHz}$ given by the covariance matrix of the Gaussian fit to obtain the result, shown in Fig. 6, we can estimate our stress resolution to be better than $500 \mathrm{kPa}$. This sensitivity is about two orders of magnitude higher than the state-of-the-art stress resolution of $25 \mathrm{MPa}$ obtained in Raman spectroscopy reported in silicon, ${ }^{39}$ which has an about 100 times higher elasticity module. With this, our relative stress resolution is comparable to the best values achieved with Raman spectroscopy. In addition, our ultrahigh stress sensitivity is accompanied by a very fine spatial resolution of about $600 \mathrm{~nm}$.

\section{Spectral dynamics}

We now examine the high-resolution spectra of single molecules under different conditions. Fig. 7a plots a Lorentzian spectrum with a FWHM of approximately $20 \mathrm{MHz}$ as expected for DBATT in bulk naphthalene. ${ }^{29}$ Roughly $60 \%$ of the molecules in the channel field with a depth of $730 \mathrm{~nm}$ show this line shape and FWHM. However, a considerable portion of the molecules undergo spectral broadening or fluctuations. Fig. $7 \mathrm{~b}-\mathrm{d}$ present three typical examples. The spectrum in Fig. $7 \mathrm{~b}$ displays a
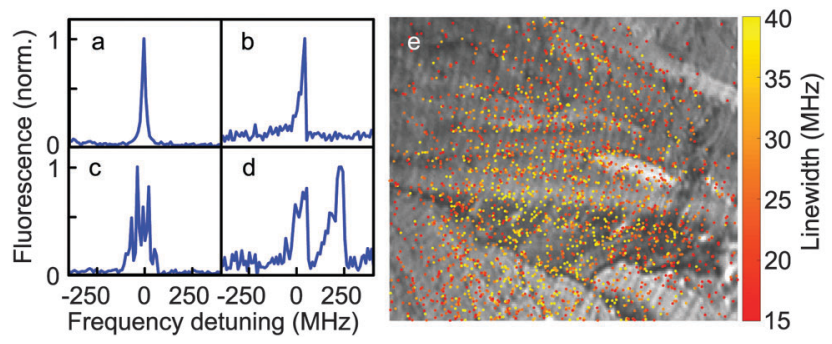

Fig. $7(a-d)$ Various fluorescence excitation line shapes of single DBATT molecules. (e) Lateral distribution of the single-molecule linewidths over the crystal field shown in Fig. $4 a$. spectral jump out of the scan range while Fig. 7c presents a molecule that undergoes fast spectral diffusion. In Fig. 7d, the molecule appears to jump twice, possibly through a photoinduced process. In Fig. 7e we plot the lateral distribution of the single-molecule linewidths over the crystal field shown in Fig. 4a. We do not find a notable correlation between the positions of the molecules and their linewidths.

In Fig. 8a-h, we present the distributions of the FWHM values obtained from Lorentzian fits to the single-molecules spectra recorded for various crystal thicknesses. We see that as the channel becomes thinner, the spread in the observed linewidths increases. This behavior hints towards an increasing fraction of defects in thinner crystals.

To model the observed behavior, we employ the approach that is commonly applied to the line shapes of single molecules observed in amorphous media such as polymers. The central hypothesis is based on the existence of spatially distributed two-level systems (TLS) with internal tunneling dynamics, which induce fluctuations due to long-range interactions with the dye molecule. ${ }^{40}$ Every TLS switches independently between
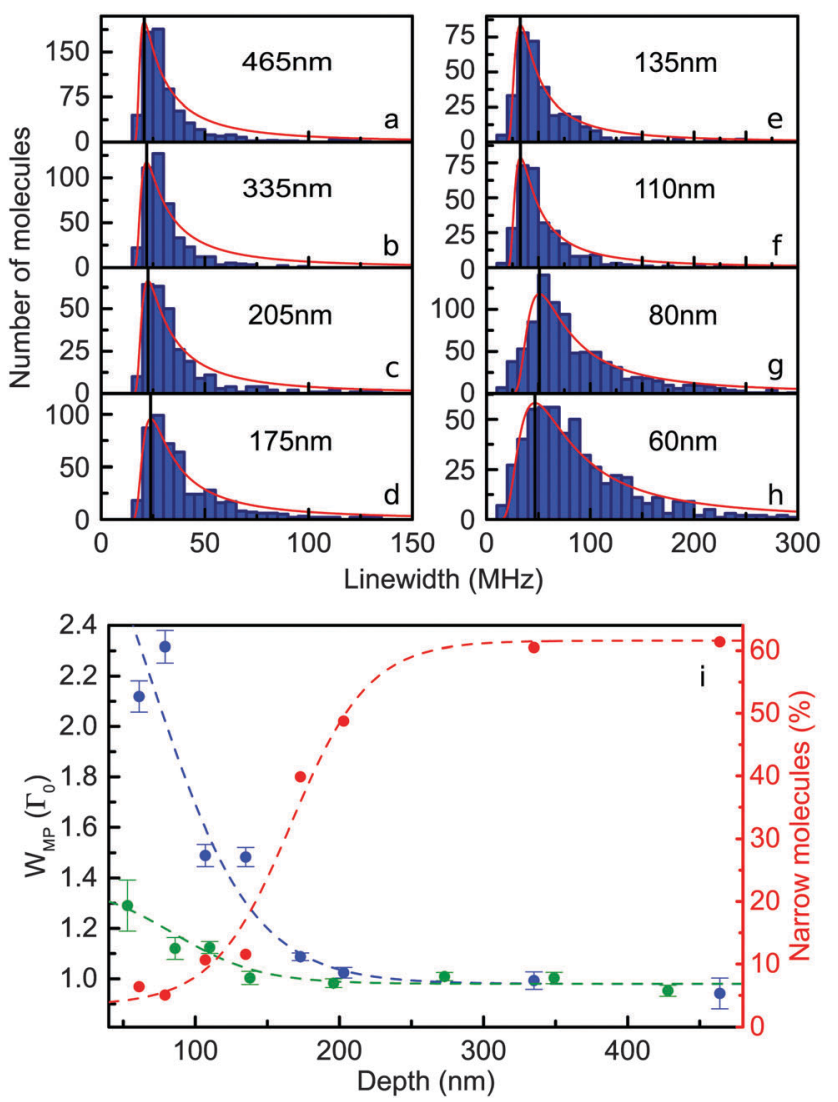

Fig. 8 (a-h) Linewidth distribution of single molecules for different naphthalene thicknesses labeled in each panel. The red curves display fits based on a modified Smirnov distribution. The black vertical lines mark the most probable width $W_{\text {MP. }}$ (i) The blue and green symbols show the variation of $W_{M P}$ normalized to the bulk linewidth $\left(\Gamma_{0}\right)$ for DBATT in naphthalene and terrylene in tetradecane, respectively. The red symbols plot the probability of finding a lifetime-limited molecule in the former system. 
two internal states, leading to a frequency shift of the probe molecule given by

$$
E(n)=\omega_{1}+\omega_{2}+\cdots+\omega_{n}
$$

after $n$ steps, where $\omega_{i}$ denotes the $i$-th energy shift after the initial state shift of a randomly chosen TLS. Within this model, the frequency fluctuation can be described as a one-dimensional random walk process that obeys Lévy statistics ${ }^{41}$ and can be described by a modified Smirnov distribution,

$$
P(W)=\frac{2 W_{0}}{\sqrt{\pi} W^{2}} \exp ^{-\left(W_{0} / W\right)^{2}} .
$$

Here, $W_{0}$ is the maximum probability value of the linewidth $W$. We remark that because of the slow decay of the distribution tail, the mean width is not an appropriate quantity for the characterization of the linewidth. In a slightly modified distribution ${ }^{42}$

$$
P(W)=\frac{W_{0}}{\sqrt{\pi} \tilde{\Gamma}(1+1 / \beta)} \frac{1}{W^{2}} \exp ^{-\left(W_{0} / W\right)^{\beta}},
$$

the most probable value of $W$ can be given by

$$
W_{\mathrm{MP}}=\left(\frac{\beta}{2}\right)^{1 / \beta} W_{0}
$$

where $\beta$ is a fit parameter and $\tilde{\Gamma}$ represents the Gamma function.

The red curves in Fig. 8 are fits according to the modified Smirnov distribution. We find that this distribution describes the observed linewidth variations very well for thin samples but less so for thicker crystals. In Fig. 8i, we plot the maximum probability $W_{\text {MP }}$ normalized to the bulk linewidth $\left(\Gamma_{0}\right)$ against the thickness of the crystals. In red, we show the probability of finding a narrow molecule, i.e., with a linewidth below $30 \mathrm{MHz}$.

To examine the applicability of our findings to other guesthost matrices, we also investigated a second system, consisting of terrylene molecules doped in tetradecane, which is known to form a polycrystalline Shpolskii matrix. ${ }^{3}$ The green symbols in Fig. 8i display the variation of the normalized $W_{\text {MP }}$ values for these measurements. While the same trend is also observed in this case, the deviation of the linewidths from their bulk value is much less pronounced. We anticipate that the degree of spectral modifications in nanoscopic media is strongly dependent on the material properties of both the host matrix and the physical boundaries.

\section{Conclusions}

We have performed a microscopic and spectroscopic study on dye molecules embedded in organic crystals under onedimensional nanoscopic confinement. By changing the size of the confinement, we have revealed a systematic transition from stable molecular transitions to spectral jump and broadening accompanied by a shift of the inhomogeneous absorption band. We have discussed these observations in the context of the strain induced in the crystal and the effect of boundaries on the crystal quality. In future work, it should also be possible to influence the interaction of the boundaries with organic crystals by treating the channel surfaces. Furthermore, one might be able to identify an optimal host matrix molecule that reduces spectral broadenings and instabilities in very narrow channels.

We have shown that a minute concentration of dye molecules such as DBATT in molecular crystals confined to nanoscopic geometries can report on the quality of the sample. The properties of thin organic films and the degree of their crystallinity ${ }^{43}$ are of current interest in molecular electronics and related fields since the intrinsic charge transport properties in organic crystals are strongly determined by the crystal structure. ${ }^{44,45}$ Crystallization under nanosized confinement has also drawn much attention in areas of pharmaceutical science, where it has been shown that geometrical confinement can strongly influence the crystallization process. ${ }^{46}$

Our findings and methodology provide interesting possibilities for new experiments that require stable single molecules in the vicinity of nanoscopic boundaries such as plasmonic antennas ${ }^{47,48}$ and subwavelength waveguides ${ }^{20}$ and for quantum nano-optical measurements that benefit from tight focusing. ${ }^{49}$ Furthermore, we believe that the agglomeration of dye molecules at crystal domain boundaries in microscopic and nanoscopic samples facilitates finding dipole-coupled molecules. ${ }^{50}$

\section{Acknowledgements}

We thank I. Harder for the fabrication of the greyscale channels. This work was supported by the European Research Council (SINGLEION) and the Alexander von Humboldt Foundation (Humboldt Professorship).

\section{References}

1 W. E. Moerner and L. Kador, Phys. Rev. Lett., 1989, 62, 2535-2538.

2 M. Orrit and J. Bernard, Phys. Rev. Lett., 1990, 65, 2716-2719.

3 T. Basche, W. E. Moerner, M. Orrit and U. Wild, Single Molecule Spectroscopy, John Wiley and Sons, 1999.

4 K. Brunner, U. Bockelmann, G. Abstreiter, M. Walther, G. Böhm, G. Tränkle and G. Weimann, Phys. Rev. Lett., 1992, 69, 3216-3219.

5 J. Y. Marzin, J. M. Gérard, A. Izraël, D. Barrier and G. Bastard, Phys. Rev. Lett., 1994, 73, 716-719.

6 A. Gruber, A. Dräbenstedt, C. Tietz, L. Fleury, J. Wrachtrup and C. v. Borczyskowski, Science, 1997, 276, 2012-2014.

7 T. Utikal, E. Eichhammer, L. Petersen, A. Renn, S. Götzinger and V. Sandoghdar, Nat. Commun., 2014, 5, 3627.

8 W. P. Ambrose and W. E. Moerner, Nature, 1991, 349, 225-227.

9 Special Issue on Spectral Dynamics and Related Topics, ed. M. Orrit, Mol. Phys., 2009, 107, 1843-1979.

10 J.-M. Segura, G. Zumofen, A. Renn, B. Hecht and U. P. Wild, Chem. Phys. Lett., 2001, 340, 77-82.

11 M. Bauer and L. Kador, J. Lumin., 2002, 98, 75-79.

12 I. Gerhardt, G. Wrigge, G. Zumofen, J. Hwang, A. Renn and V. Sandoghdar, Phys. Rev. A: At., Mol., Opt. Phys., 2009, 79, 011402(R). 
13 I. Gerhardt, G. Wrigge, M. Agio, P. Bushev, G. Zumofen and V. Sandoghdar, Opt. Lett., 2007, 32, 1420-1422.

14 F. Kulzer, S. Kummer, R. Matzke, C. Bräuchle and T. Basché, Nature, 1997, 387, 688-691.

15 E. Geva and J. L. Skinner, J. Phys. Chem. B, 1997, 101, 8920-8932.

16 Z. M. Wang, Self-Assembled Quantum Dots, Springer Science \& Business Media, 2007, vol. 1.

17 R. S. Meltzer, W. M. Yen, H. Zheng, S. P. Feofilov, M. J. Dejneka, B. M. Tissue and H. B. Yuan, Phys. Rev. B: Condens. Matter Mater. Phys., 2001, 64, 100201.

18 K. Mawatari, T. Tsukahara, Y. Tanaka, Y. Kazoe, P. Dextras and T. Kitamori, Extended-Nanofluidic Systems for Chemistry and Biotechnology, Imperial College Press, London, 2012.

19 A. Plößl and G. Kräuter, Mater. Sci. Eng., R, 1999, 25, 1-88.

20 S. Faez, P. Türschmann, H. R. Haakh, S. Götzinger and V. Sandoghdar, Phys. Rev. Lett., 2014, 113, 213601.

21 A. Kochendörfer, Z. Kristallogr. - Cryst. Mater., 1937, 97, 263-299.

22 R. B. Gordon, Acta Metall., 1965, 13, 199-203.

23 F. C. Frank and W. T. Read, Phys. Rev., 1950, 79, 722-723.

24 G. Meurant, Adv. Appl. Mech., Elsevier Science, 1983.

25 S. Weisenburger and V. Sandoghdar, Contemp. Phys., 2015, 56, 123-143.

26 M. Matsushita, A. Bloeß, Y. Durand, J. Y. P. Butter, J. Schmidt and E. J. J. Groenen, J. Chem. Phys., 2002, 117, 3383-3390.

27 A. Bloeß, Y. Durand, M. Matsushita, H. Van Dermeer, G. J. Brakenhoff and J. Schmidt, J. Microsc., 2002, 205, 76-85.

28 A. V. Naumov, A. A. Gorshelev, Y. G. Vainer, L. Kador and J. Köhler, Angew. Chem., Int. Ed., 2009, 48, 9747-9750.

29 F. Jelezko, B. Lounis and M. Orrit, J. Chem. Phys., 1997, 107, 1692-1702.

30 P. Siyushev, G. Stein, J. Wrachtrup and I. Gerhardt, Nature, 2014, 509, 66-70.

31 M. Croci, H.-J. Müschenborn, F. Güttler, A. Renn and U. P. Wild, Chem. Phys. Lett., 1993, 212, 71-77.
32 S. Kummer, T. Basche and C. Bräuchle, Chem. Phys. Lett., 1994, 229, 309-316.

33 A. Müller, W. Richter and L. Kador, Chem. Phys. Lett., 1995, 241, 547-554.

34 T. Sesselmann, W. Richter, D. Haarer and H. Morawitz, Phys. Rev. B: Condens. Matter Mater. Phys., 1987, 36, 7601-7611.

35 P. F. Jones, J. Chem. Phys., 1968, 48, 5448-5456.

36 C. P. Brock and J. D. Dunitz, Acta Crystallogr., Sect. B: Struct. Crystallogr. Cryst. Chem., 1982, 38, 2218-2228.

37 V. K. Jindal and J. Kalus, Phys. Status Solidi B, 1986, 133, 89-99.

38 J. F. J. Jordan, A. Axmann, H. Egger and J. Kalus, Phys. Status Solidi A, 1982, 71, 457-462.

39 I. D. Wolf, Semicond. Sci. Technol., 1996, 11, 139.

40 P. d. Reilly and J. L. Skinner, Phys. Rev. Lett., 1993, 71, 4257-4260.

41 G. Zumofen and J. Klafter, Chem. Phys. Lett., 1994, 219, 303-309.

42 A. Walser, G. Zumofen, A. Renn, S. Götzinger and V. Sandoghdar, Mol. Phys., 2009, 107, 1897-1909.

43 S. Schiefer, M. Huth, A. Dobrinevski and B. Nickel, J. Am. Chem. Soc., 2007, 129, 10316-10317.

44 A. Troisi and G. Orlandi, J. Phys. Chem. B, 2005, 109, 1849-1856.

45 A. Troisi and G. Orlandi, Phys. Rev. Lett., 2006, 96, 086601.

46 Q. Jiang and M. D. Ward, Chem. Soc. Rev., 2014, 43, 2066-2079.

47 S. Kühn, U. Håkanson, L. Rogobete and V. Sandoghdar, Phys. Rev. Lett., 2006, 97, 017402.

48 B. Hoffmann, S. Vassant, X.-W. Chen, S. Götzinger, V. Sandoghdar and S. Christiansen, Nanotechnology, 2015, 26, 404001.

49 A. Maser, B. Gmeiner, T. Utikal, S. Götzinger and V. Sandoghdar, Nat. Photonics, 2016, DOI: 10.1038/nphoton.2016.63.

50 C. Hettich, C. Schmitt, J. Zitzmann, S. Kühn, I. Gerhardt and V. Sandoghdar, Science, 2002, 298, 385. 